\title{
Dissecting graph measure performance for node clustering in LFR parameter space
}

\author{
Vladimir Ivashkin ${ }^{1}$ and Pavel Chebotarev ${ }^{2}$ \\ 1 Moscow Institute of Physics and Technology, Moscow, Russia, \\ vladimir.ivashkin@phystech.edu \\ 2 Institute of Control Sciences of RAS, Moscow, Russia, \\ pavel4e@gmail.com
}

\begin{abstract}
Graph measures that express closeness or distance between nodes can be employed for graph nodes clustering using metric clustering algorithms. There are numerous measures applicable to this task, and which one performs better is an open question. We study the performance of 25 graph measures on generated graphs with different parameters. While usually measure comparisons are limited to general measure ranking on a particular dataset, we aim to explore the performance of various measures depending on graph features. Using an LFR graph generator, we create a dataset of 11780 graphs covering the whole LFR parameter space. For each graph, we assess the quality of clustering with $k$-means algorithm for each considered measure. Based on this, we determine the best measure for each area of the parameter space. We find that the parameter space consists of distinct zones where one particular measure is the best. We analyze the geometry of the resulting zones and describe it with simple criteria. Given particular graph parameters, this allows us to recommend a particular measure to use for clustering.
\end{abstract}

Keywords: graphs, graph measures, clustering, kernel k-means

\section{Introduction}

Graph nodes clustering is one of the central tasks in graph structure analysis. It provides a partition of nodes into disjoint clusters (communities), which are groups of nodes that are characterized by strong mutual connections or similar external connections. It can be of practical use for graphs representing reallife systems, such as social networks or industrial processes. Clustering allows to infer some information about the system: the nodes of the same cluster are highly similar, while the nodes of different clusters are dissimilar. The technique can be applied without any labeled data to extract important insights about a network.

There are different approaches to clustering, including ones based on modularity optimization 446, label propagation algorithm 485, Markov cluster process [52 17], and spectral clustering [53. In this work, we use an approach based on choosing a closeness measure on a graph, which allows one to use any metric clustering algorithm (e.g., [55]). 
The choice of the measure significantly affects the quality of clustering. Classical measures are the Shortest Path [8] and the Commute Time [26 distances. The former is the minimum number of edges in a path between a given pair of nodes. The latter is the expected number of steps from one node to the other and back in a random walk on the graph. There is a number of other measures, including recent ones (e.g., 20133]); many of them are parametric. Despite the fact that graph measures are compatible with any metric algorithm, in this paper, we restrict ourselves to the kernel $k$-means algorithm (e.g., 24]).

We base our research on a generated set of graphs. There are various algorithms to generate graphs with community structures. The well-known ones are the Stochastic Block Model [30] and Lancichinetti-Fortunato-Radicchi benchmark 38 (hereafter, LFR). The first one is an extension of the Erdős-Rényi model with different intra- and intercluster probabilities of edge creation. The second one involves power law distributions of node degrees and community sizes. There are other generation models, e.g., Naive Scale-free Clustering [46]. We choose the LFR model: although it misses some key properties of real graphs, like diameter or the clustering coefficient, this model has been proven to be effective in meta-learning 47 .

There are many measure benchmarking studies considering node classification and clustering for both generated graphs and real-world datasets, including 23/50|51|2 32 29|28|3|4 16 39. Despite a large number of experimental results, an exact theory is still a matter of the future. One of the most interesting theoretical results on graph measures is [41, where some unattractive features of the Commute Time distance on large graphs were explained theoretically, and a reasonable amendment was proposed to fix the problem. Beyond the complexity of such proofs, there is still very little empirical understanding of what effects need to be proven. Our empirical work has two main differences from the previous ones. First, we consider a large number of graph measures, which for the first time gives a fairly complete picture. Second, unlike the previous studies aimed at revealing the global leaderboard, we are looking for the leading measures for each set of the LFR parameters.

We aim to explore the performance of of the 25 most popular measures in the graph clustering problem on a set of generated graphs with different parameters. We assess the quality of clustering with every considered measure and determine the best measure for every region of the graph parameter space.

Our contributions are as follows:

- We generate a dataset of 11780 graphs covering the entire parameter space of the LFR generator;

- We consider a broad set of measures and rank them by clustering performance on this dataset;

- We determine the graph features that are responsible for measure leadership;

- We find the regions of each measure's leadership in the graph parameter space.

Our framework for clustering with graph measures as well as the collected dataset are available at https://github.com/vlivashkin/pygkernels. 


\section{Definitions}

\section{$2.1 \quad$ Kernel $k$-means}

The original $k$-means algorithm [40]42] clusters objects in Euclidean space. It requires coordinates of the objects to determine the distances between them and centroids. The algorithm can be generalized to use the degree of closeness between the objects without defining a particular space. This technique is called the kernel trick, usually it is used to bring non-linearity to linear algorithms. The algorithm that uses the kernel trick is called kernel k-means (see, e.g., 24]). For graph node clustering scenario, we can use graph measures as kernels for the kernel $k$-means.

Initially, the number of clusters is known and we need to set the initial state of centroids. The results of clustering with $k$-means are very sensitive to the initial state. Usually, the algorithm runs several times with different initial states (trials) and chooses the "best" trial. There are different approaches to the initialization; we consider three of them: random data points, $k$-means ++ [1], and random partition. We combine all these strategies to reduce the impact of the initialization strategy on the result.

\subsection{Closeness measures}

For a given graph $G, V(G)$ is the set of its vertices and $A$ is its adjacency matrix. A measure on $G$ is a function $\kappa: V(G) \times V(G) \rightarrow \mathbb{R}$, which gets two nodes and returns closeness (larger means closer) or distance (larger means farther).

A kernel on a graph is a graph nodes' closeness measure that has an inner product representation. Any symmetric positive semidefinite matrix is an inner product matrix (also called Gram matrix). A kernel matrix $K$ is a square matrix that contains similarities for all pairs of nodes in a graph.

To use kernel $k$-means, we need kernels. Despite that not all closeness measures we consider are Gram matrices, we treat them as kernels. The applicability of this approach was confirmed in 24. For the list of measures below, we use the word "kernel" only for the measures that satisfy the precise definition of kernel.

Classical measures Shortest Path distance 8 (SP) and Commute Time distance 26] (CT) are defined as distances, so we need to transform them into similarities to use as kernels. We apply the following distance to closeness transformation [117]:

$$
K=-H \mathcal{D} H ; H=I-E / n,
$$

where $\mathcal{D}$ is a distance matrix, $E$ is the matrix of ones, $I$ is the identity matrix, and $n$ is the number of nodes.

In this paper, we examine 25 graph measures (or, more exactly, 25 parametric families of measures). We present these measures grouped by type similarly to [2]:

- Adjacency Matrix $A$ based kernels and measures.

- Katz kernel: $K_{\alpha}^{\mathrm{Katz}}=(I-\alpha A)^{-1}, 0<\alpha<\rho^{-1}$, where $\rho$ is the spectral radius of $A$ [35] (also known as Walk proximity [12] or von Neumann diffusion kernel [34 49]). 
- Communicability kernel $K_{t}^{\mathrm{Comm}}=\operatorname{expm}(t A), t>0$, where expm means matrix exponential 251819].

- Double Factorial closeness: $K_{t}^{\mathrm{DF}}=\sum_{k=0}^{\infty} \frac{t^{k}}{k ! !} A^{k}, t>0$ [20].

- Laplacian Matrix $L=D-A$ based kernels and measures, where $D=\operatorname{Diag}(A$.

$\mathbf{1}$ ) is the degree matrix of $G, \operatorname{Diag}(\mathbf{x})$ is the diagonal matrix with vector $\mathbf{x}$ on the main diagonal.

- Forest kernel: $K_{t}^{\text {For }}=(I+t L)^{-1}, t>0$ (also known as Regularized Laplacian kernel) 10.

- Heat kernel: $K_{t}^{\text {Heat }}=\operatorname{expm}(-t L), t>0$ [14.

- Normalized Heat kernel: $K_{t}^{\mathrm{NHeat}}=\operatorname{expm}(-t \mathcal{L}), \mathcal{L}=D^{-\frac{1}{2}} L D^{-\frac{1}{2}}$, $t>0[15$.

- Absorption kernel: $K_{t}^{\mathrm{Abs}}=(t A+L)^{-1}, t>033$.

- Markov Matrix $P=D^{-1} A$ based kernels and measures.

- Personalized PageRank closeness: $K_{\alpha}^{\mathrm{PPR}}=(I-\alpha P)^{-1}$, $0<\alpha<1$ [5].

- Modified Personalized PageRank: $K_{\alpha}^{\mathrm{MPPR}}=(I-\alpha P)^{-1} D^{-1}=(D-$ $\alpha A)^{-1}, 0<\alpha<1$ [36].

- PageRank heat closeness: $K_{t}^{\mathrm{HPR}}=\operatorname{expm}(-t(I-P)), t>0$ [13].

- Randomized Shortest Path distance. Using $P$ and the matrix of the SP distances $C$ first get $Z[56]$ :

$$
Z=(I-W)^{-1}, \text { where } W=P \circ \exp (-\beta C) .
$$

Then $S=(Z(C \circ W) Z) \div Z ; \bar{C}=S-\mathbf{e} \operatorname{diag}(S)^{T}$, and finally, $\mathcal{D}_{\mathrm{RSP}}=$ $\left(\bar{C}+\bar{C}^{T}\right) / 2$. Here $\circ$ and $\div$ are element-wise multiplication and division, $\operatorname{diag}(S)$ is the column vector of the diagonal elements of $S$. The kernel version $K^{\mathrm{RSP}}(t)$ can be obtained with (1).

- Free Energy distance. Using $Z$ from (2) : $\widetilde{Z}=Z \operatorname{Diag}(Z)^{-1} ; \Phi=-1 / \beta \log \widetilde{Z}$; $\mathcal{D}_{\mathrm{FE}}=\left(\Phi+\Phi^{T}\right) / 2$ [37. Kernel version $K^{\mathrm{FE}}(t)$ can be obtained with (11).

- Sigmoid Commute Time kernels.

- Sigmoid Commute Time kernel:

$$
K_{t}^{\mathrm{SCT}}=\sigma\left(-t K^{\mathrm{CT}} / \operatorname{std}\left(K^{\mathrm{CT}}\right)\right), t>0,
$$

where $K^{\mathrm{CT}}$ is the matrix of Commute Time proximity, std is the standard deviation of all elements of a matrix, $\sigma$ is the element-wise sigmoid function $\sigma(x)=1 /\left(1+e^{-x}\right)$ [54].

- Sigmoid Corrected Commute Time kernel. First of all, we need the Corrected Commute Time kernel [41]: $K^{\mathrm{CCT}}=H D^{-\frac{1}{2}} M(I-M)^{-1} M D^{-\frac{1}{2}} H$, where $M=D^{-\frac{1}{2}}\left(A-\frac{\overrightarrow{\mathbf{d}} \overrightarrow{\mathbf{d}}^{T}}{\operatorname{vol}(G)}\right) D^{-\frac{1}{2}}, H$ is the centering matrix $H=$ $I-E / n, \overrightarrow{\mathbf{d}}$ is the vector of diagonal elements of $D$ and $\operatorname{vol}(G)$ is the sum of all elements of $A$. Then, apply (3) replacing $K^{\mathrm{CT}}$ with $K^{\mathrm{CCT}}$ to obtain $K^{\mathrm{SCCT}}$

Occasionally, element-wise logarithm is applied to the resulting kernel matrix 932. We apply it to almost all investigated measures and consider the 
resulting measures separately from their plain versions (see Table 1). For some measures, like Forest kernel, this is well-known practice [9], while for others, like Double Factorial closeness, this transformation, to the best of our knowledge, is applied for the first time. The considered measures and their short names are summarized in Table 1

Table 1: Short names of considered kernels and other measures.

\begin{tabular}{|c|c|c|c|}
\hline Family & \begin{tabular}{|l|}
\multicolumn{2}{|c}{ Shor } \\
Plain
\end{tabular} & $\begin{array}{l}\text { rt name } \\
\text { |Log }\end{array}$ & Full name \\
\hline \multirow{3}{*}{$\begin{array}{l}\text { Adjacency } \\
\text { matrix based } \\
\text { kernels }\end{array}$} & Katz & $\operatorname{logKatz}$ & Katz kernel \\
\hline & Comm & $\log$ Comm & Communicability kernel \\
\hline & DF & $\log \mathrm{DF}$ & Double Factorial closeness \\
\hline \multirow{4}{*}{$\begin{array}{l}\text { Laplacian based } \\
\text { kernels }\end{array}$} & For & $\log$ For & Forest kernel \\
\hline & Heat & $\operatorname{logHeat}$ & Heat kernel \\
\hline & NHeat & $\log N H e a t$ & Normalized Heat kernel \\
\hline & Abs & $\log \mathrm{Abs}$ & Absorption kernel \\
\hline \multirow{5}{*}{$\begin{array}{l}\text { Markov matrix } \\
\text { based kernels } \\
\text { and measures }\end{array}$} & PPR & $\log P P R$ & Personalized PageRank closeness \\
\hline & MPPR & $\log M P P R$ & Modified Personalized PageRank \\
\hline & HPR & $\log H P R$ & PageRank heat closeness \\
\hline & $\mathrm{RSP}$ & - & Randomized Shortest Path kernel \\
\hline & $\mathrm{FE}$ & - & Free Energy kernel \\
\hline Sigmoid & $\mathrm{SCT}$ & - & Sigmoid Commute Time kernel \\
\hline \multirow[t]{2}{*}{ Commute Time } & SCCT & - & Sigmoid Corrected Commute Time kernel \\
\hline & $\mathrm{SP}-\mathrm{CT}$ & - & Linear combination of SP and CT \\
\hline
\end{tabular}

\section{Dataset}

We collected a paired dataset of graphs and the corresponding results of clustering with each measure mentioned in Table 1 In this section, we describe the graph generator, the sampling strategy, the graph characteristic features, and the pipeline for the measure score calculation.

We use Lancichinetti-Fortunato-Radicchi (LFR) graph generator. It generates non-weighted graphs with ground truth non-overlapping communities. The model has five mandatory parameters: the number of nodes $n(n>0)$, the power law exponent for the degree distribution $\tau_{1}\left(\tau_{1}>1\right)$, the power law exponent for the community size distribution $\tau_{2}\left(\tau_{2}>1\right)$, the fraction of intracommunity edges incident to each node $\mu(0 \leq \mu \leq 1)$, and either minimum degree (min degree) or average degree (avg degree). There are also several extra parameters: maximum degree (max degree), minimum community size (min community), maximum community size (max community). Not the whole LFR parameter space corresponds to common real-world graphs; most of such graphs are described with $\tau_{1} \in[1,4]$ and $\mu<0.5$ (e.g., [22]). However, there is also an interesting case of bipartite/multipartite-like graphs with $\mu>0.5$. Our choice 
is to consider the entire parameter space to cover all theoretical and practical cases.

For the generation, we consider $10<n<1500$. It is impossible to generate a dataset with a uniform distribution of all LFR parameters, because $\tau_{1}$ and $\tau_{2}$ parameters are located on rays. We transform $\tau_{1}$ and $\tau_{2}$ to $\tilde{\tau}_{i}=1-\left(1 / \tau_{i}^{0.7}\right), i=$ 1,2 to bring their scope to the $[0,1]$ interval. In this case, "realistic" settings with $\tau_{1} \in[1,4]$ take up $62 \%$ of the variable range. Also, as avg degree feature is limited by the number of nodes $n$ of a particular graph, we decided to replace it with density (avg degree/( $n-1)$ ). It belongs to $[0,1]$. Using all these considerations, we collected our dataset by uniformly sampling parameters for LFR generator from the set $\left\{n, \tilde{\tau}_{1}, \tilde{\tau}_{2}, \mu\right.$, density $\}$ and generating graphs with these parameters. Additionally, we filter out all disconnected graphs.

In total, we generated 11780 graphs. It is worth noting that the generator fails for some sets of parameters, so the resulting dataset is not uniform (see Fig. (1). In our study, non-uniformity is not a critical issue, because we are interested in local effects, rather than global leadership. Moreover, true uniformity for LFR parameter space is impossible, due to the unlimited scope of parameters.
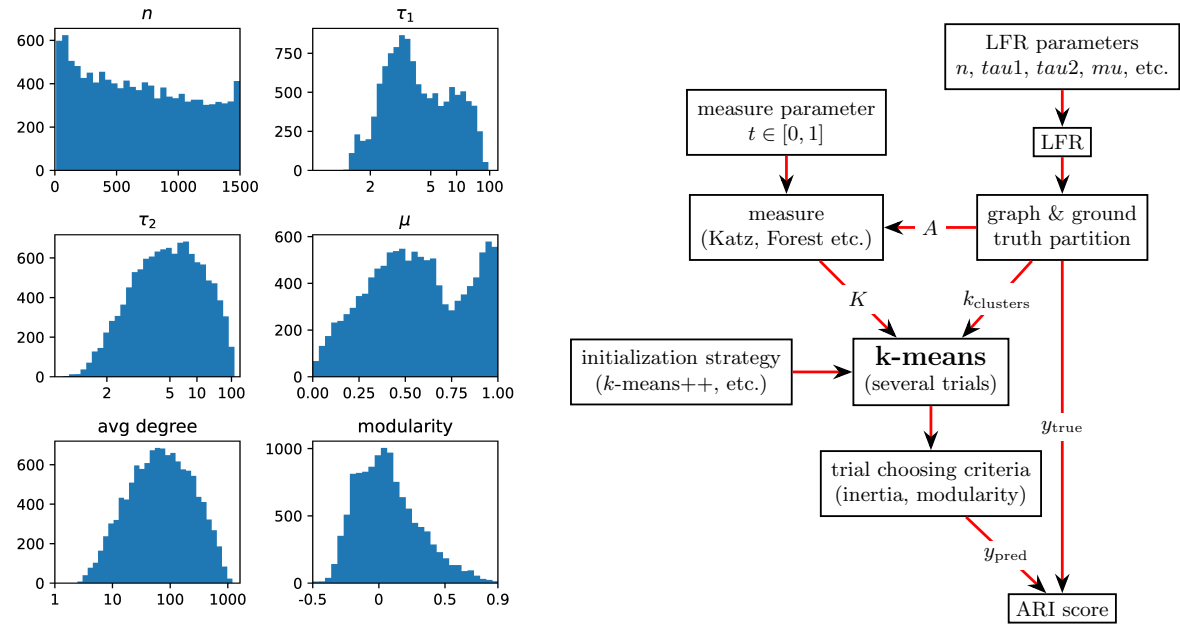

Fig. 1: Distribution of graph features in the dataset

Fig. 2: Measuring ARI clustering score for a particular graph, measure, and measure parameter

For our research, we choose a minimum set of the features that describe particular properties of graphs and are not interchangeable.

The LFR parameters can be divided into three groups by the graph properties they reflect:

- The size of the graph and the communities: $n, \tau_{1}$, min community, max community; 
- The density and uniformity of the node degree distribution: $\tau_{2}$, min degree, avg degree, max degree. As avg degree depends on $n$, it is distributed exponentially, so we use $\log$ (avg degree) instead;

- The cluster separability: $\mu$. As $\mu$ parameter considers only the ratio between the number of inter-cluster edges and the number of nodes but ignores overall density, we use modularity [44 as a more appropriate measure for cluster separability.

Thus, the defined set of features $\left\{n, \tau_{1}, \tau_{2}\right.$, avg degree, modularity $\}$ is enough to consider all graph properties mentioned above. Although modularity is a widely used measure, it suffers from resolution limit problems 21. We acknowledge that this may cause some limitations in our approach, which should be the topic of further research.

For every generated graph, we calculate the top ARI score for every measure 31. The popular NMI clustering score is known to be biased towards smaller clusters, according to [27. We choose ARI as a clustering score that is both well known and unbiased. As soon as every measure has a parameter, we perform clustering for a range of parameter values (we transform the parameter to become in the $[0,1]$ interval and then choose 16 values linearly spaced from 0 to 1 ). For each value, we run $6+6+6$ trials of $k$-means ( 6 trials for each of three initialization methods).

Fig. 2 shows the pipeline we use to calculate ARI score for a given LFR parameter set, a measure, and a measure parameter. Measure parameters are not the subject of our experiments, so for every measure we just take the result of the measure with the value of the parameter that gives the best ARI score.

Because of the need to iterate over graphs, measures, parameter values, and initializations, the task is quite computationally complex. The total computation time was 20 days on $18 \mathrm{CPU}$ cores and 6 GPUs.

\section{Results}

\subsection{Global leadership in LFR space}

As a rough estimate of the measures' applicability, we calculate global leadership on our generated dataset. We divide the dataset into parts corresponding to two different cases of clustering: associative (graphs with modularity $\geq 0$ on the ground truth partition) and the complementary dissociative case. The negative modularity case basically corresponds to the $\mu>0.5$ setup of LFR generator.

We rank the measures by their ARI score on every graph of the dataset. The aggregated rank is defined as the position of the measure in this list, averaged over the dataset (see Tables 2a and2b; smaller rank is better). It is important to note that the global leadership does not give a comprehensive advice on which measure is better to use, because for a particular graph, the global leader can perform worse than the local winner. Here, we consider the entire LFR space, not just its zone corresponding to common real-world graphs, so the ranking may differ from those obtained for restricted settings. 
Table 2: Leaderboards for associative and dissociative cases. The ARI column shows the mean ARI across the dataset.

\begin{tabular}{|c|c|c|}
\hline \# Measure & \multicolumn{2}{|c|}{ Rank Wins, \% ARI } \\
\hline $1 \mathrm{RSP}$ & 4.1 & 40.00 .67 \\
\hline $2 \mathrm{SCCT}$ & 5.1 & 50.50 .68 \\
\hline $3 \log$ NHeat & 5.3 & 34.70 .66 \\
\hline $4 \operatorname{logHeatPR}$ & 5.3 & 34.90 .66 \\
\hline $5 \mathrm{FE}$ & 5.5 & 35.80 .66 \\
\hline 6 logKatz & 5.5 & 39.50 .66 \\
\hline $7 \log P P R$ & 6.2 & 35.10 .65 \\
\hline $8 \log$ Comm & 6.3 & $40.5 \quad 0.64$ \\
\hline 9 logModifPPR & 6.5 & 34.40 .65 \\
\hline $10 \mathrm{SCT}$ & 7.3 & 36.10 .64 \\
\hline 11 SP-CT & 7.5 & 32.60 .64 \\
\hline $12 \log \mathrm{Abs}$ & 8.1 & 33.80 .63 \\
\hline $13 \operatorname{logFor}$ & 8.8 & 33.80 .60 \\
\hline $14 \log$ Heat & 9.2 & 31.10 .58 \\
\hline 15 NHeat & 9.6 & 35.10 .56 \\
\hline 16 HeatPR & 10.3 & 32.20 .59 \\
\hline 17 Comm & 11.5 & 26.40 .52 \\
\hline $18 \log \mathrm{DF}$ & 12.4 & 22.70 .46 \\
\hline 19 Heat & 13.7 & 27.20 .46 \\
\hline 20 Katz & 15.2 & 10.10 .43 \\
\hline $21 \mathrm{DF}$ & 16.0 & $\begin{array}{ll}12.1 & 0.37\end{array}$ \\
\hline $22 \mathrm{PPR}$ & 17.8 & 11.10 .35 \\
\hline 23 For & 20.4 & 7.90 .19 \\
\hline $24 \mathrm{Abs}$ & 21.1 & 7.10 .16 \\
\hline 25 ModifPPR & 22.1 & 4.70 .12 \\
\hline
\end{tabular}

(a) Associative graphs. The win percentage is calculated among 6777 graphs in the dataset.

\begin{tabular}{lrrr}
\hline \# Measure & Rank Wins, \% ARI \\
\hline 1 SCCT & 3.7 & 63.9 & 0.70 \\
2 RSP & 7.0 & 18.6 & 0.46 \\
3 SP-CT & 8.1 & 16.6 & 0.45 \\
4 SCT & 8.1 & 14.5 & 0.46 \\
5 NHeat & 8.5 & 10.1 & 0.40 \\
6 logHeatPR & 8.6 & 14.5 & 0.41 \\
7 FE & 8.9 & 15.5 & 0.43 \\
8 logNHeat & 9.0 & 13.9 & 0.39 \\
9 logPPR & 9.4 & 13.8 & 0.39 \\
10 Katz & 9.8 & 2.8 & 0.34 \\
11 Comm & 10.3 & 5.2 & 0.33 \\
12 logModifPPR & 10.8 & 13.5 & 0.37 \\
13 logKatz & 10.9 & 14.4 & 0.37 \\
14 Abs & 11.0 & 13.3 & 0.35 \\
15 DF & 12.2 & 2.6 & 0.27 \\
16 logAbs & 12.8 & 12.1 & 0.35 \\
17 HeatPR & 15.9 & 0.6 & 0.16 \\
18 logFor & 15.9 & 5.3 & 0.22 \\
19 Heat & 15.9 & 0.7 & 0.09 \\
20 PPR & 16.4 & 0.5 & 0.15 \\
21 logHeat & 16.6 & 0.7 & 0.10 \\
22 logDF & 18.1 & 3.7 & 0.08 \\
23 logComm & 18.2 & 0.7 & 0.07 \\
24 For & 21.4 & 0.0 & 0.02 \\
25 ModifPPR & 21.7 & 0.1 & 0.02 \\
\hline
\end{tabular}

(b) Dissociative graphs. The win percentage is calculated among 5003 graphs in the dataset.

Table 2a shows that there are several leading measures whose quality is not much different. The best measures are RSP (by rank) and SCCT (by the number of wins and the mean ARI). Dissociative case has an undisputed leader, SCCT (Table 2b).

The above division into two cases does not exhaust all the variety of graphs. For the further more precise study of the measures' applicability, we will look for the leadership zones of each measure.

\subsection{Feature importance study}

First of all, we find out which graph features among the LFR parameters are important for the choice of the best measure and which are not. To do that, we use Linear Discriminant Analysis [43] (LDA). This method finds a new basis in 
the feature space to classify a dataset in the best way. It also shows how many components of basis are required to fit the majority of data.

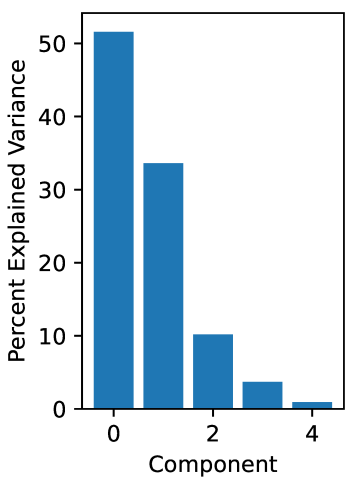

(a) Explained variance.

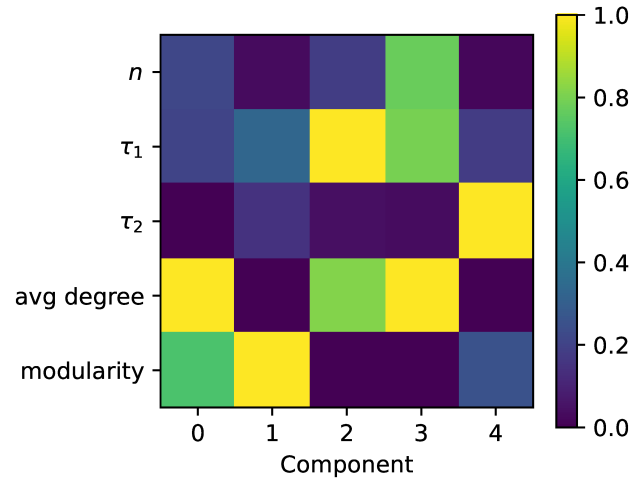

(b) Features' contribution to LDA components.

Fig. 3: The results of LDA analysis.

Fig. 3a shows that the first two components account for about $90 \%$ of the explained variance. Fig. 3b shows that these components include only $\tau_{1}$, avg degree, and modularity. The fact that $n$ is not included means that the size of the graph as well as the density are not of primary importance for choosing the best measure. So is not $\tau_{2}$ measuring the diversity of cluster sizes.

To detect the zones of measure leadership, we need to know the leadership on average in each area of space rather than the wins in particular points. To determine the local measure leadership, we need to introduce a filtering algorithm that for every point of the space returns the leading measure depending on the closest data points. As the choice of measure is mainly dependent on three features $\left\{\tau_{1}\right.$, avg degree, modularity $\}$, we can limit our feature space to them.

\subsection{Gaussian filter in feature space}

Using a filter in the feature space, we can suppress the noise and reveal the actual zones of leadership for the measures. We use the Gaussian filter with a scale parameter $\sigma$. For every given point of the space, it takes the data points that are closer than $3 \sigma$ and averages ARIs of the chosen points with a weight $e^{- \text {dist }^{2} / 2 \sigma^{2}}$. This allows to give larger weights to closer points. If there are less than three data points inside the sphere with a $3 \sigma$ radius, the filter returns nothing, allowing to ignore the points with insufficient data in their vicinity.

Before applying the filter, we prepare the dataset. First, we isolate the case when several measures reach ARI $=1$ into a separate measure called "several". Also, we normalize the standard deviation of every feature distribution to one. 
To choose $\sigma$, we apply the filter with different values of $\sigma$ and look at the number of connected components in the feature space. The needed $\sigma$ should be large enough to suppress the noise, however, it should not suppress small zones. Guided by this heuristic, we choose $\sigma=0.6$.

Table 3: The leaderboard of measure wins after filtering with $\sigma=0.6$

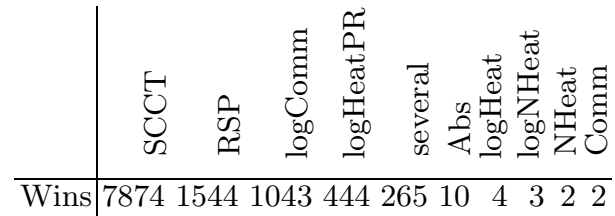

After filtering with $\sigma=0.6$, the leaderboard of measure wins changes (see Table 3). Only four measures keep their positions: SCCT, RSP, logComm, and $\log$ HeatPR. There is also a special case of several winning measures (named "several"), when the predicted partition reaches ARI $=1$ for several measures. The presence of several winners makes it difficult to analyze zones of measure's leadership, so we decided to exclude this case from the detailed analysis in this work. Filtering shows that these four measures do have zones of leadership, otherwise they would be filtered out. We can plot the entire feature space colored by the leadership zones of the measures (see Fig. 4). As the resulting space is $3 \mathrm{D}$, we indicate its slices by their coordinates.

The zones of measure leadership can be described by the following approximate criteria:

- SCCT: in many domains of the parameter space;

- RSP: $\tau_{1}$ up to 5, modularity in $-0.25 . .0 .3$;

- logComm: modularity in 0..0.3, avg degree up to 100;

- logHeatPR: modularity above 0.3, avg degree up to 50;

- several: high modularity or high avg degree.

\section{Conclusions}

In this work, we have shown that the global leadership of measures does not provide comprehensive knowledge about graph measure performance in clustering tasks. We demonstrated that among 25 measures, SCCT is the best measure for the LFR graphs both by winning rate and ranking. However, there are also smaller distinct zones of leadership for RSP, logComm, and logHeatPR. Other measures, including those with high rank, fail to form their leadership zones.

Our results do not contradict those of other experimental works and, moreover, refine them by providing new findings. LogComm was first introduced in 32 and won in the competitions on graphs generated with a particular set of SBM parameters. This study confirms its leadership, but only for a certain type 


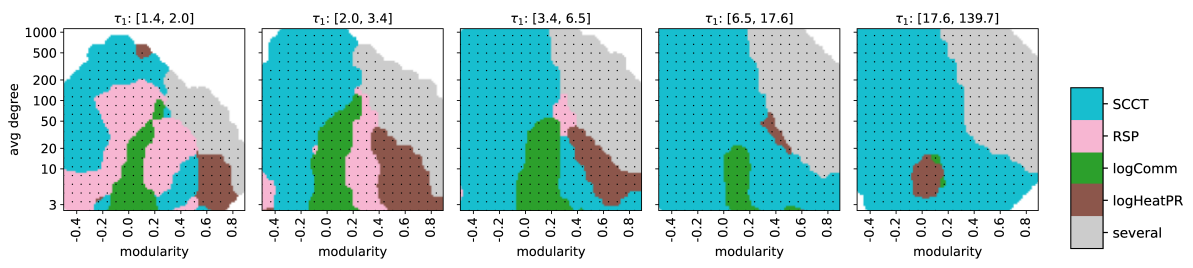

(a) Slices by $\tau_{1}$.
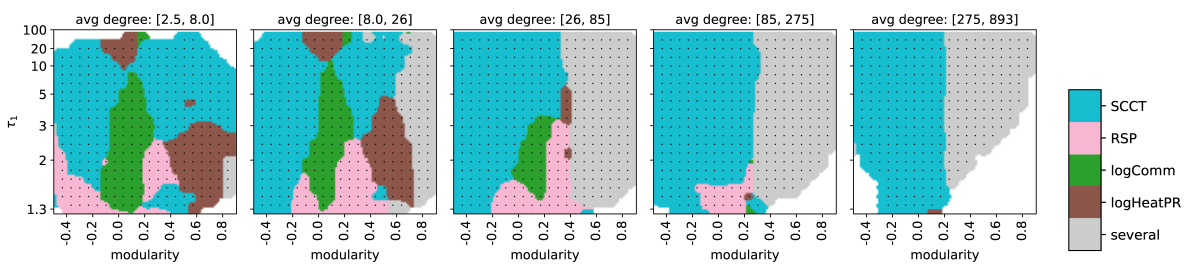

(b) Slices by avg degree.
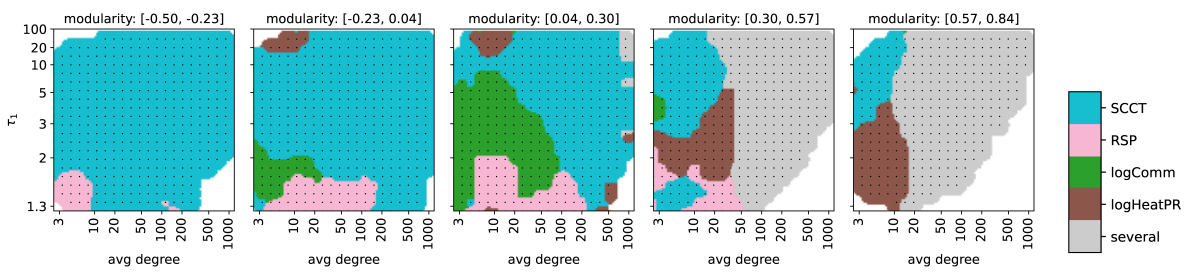

(c) Slices by modularity.

Fig. 4: The feature space $\left\{\tau_{1}\right.$, avg degree, modularity $\}$ divided into the leadership zones of six measures.

of graphs. Another interesting finding is $\operatorname{logHeatPR}$, which shows unexpectedly good performance within its zone of leadership.

Accoring to LDA analysis results, the leadership of measure is determined mainly by $\left\{\tau_{1}\right.$, avg degree, modularity $\}$. One of the interesting consequences is that the leadership does not depend on $n$. This effect could be caused by the fact that we limited the size of the graphs to $n<1500$. It is not guaranteed to be preserved for large graphs.

This study is based on the LFR benchmark data. More research is needed to determine how well the results for LFR fit with to real-world. This would assess the applicability of our findings to practical cases.

It should be noted that our study is insensitive to the non-uniformity of the generated dataset. While manipulations with this dataset may affect the global leaderboard, they cannot change the local leadership, which is the focus of the present work. 


\section{References}

1. Arthur, D., Vassilvitskii, S.: k-means++: The advantages of careful seeding. Tech. rep., Stanford University (2006)

2. Avrachenkov, K., Chebotarev, P., Rubanov, D.: Kernels on graphs as proximity measures. In: International Workshop on Algorithms and Models for the WebGraph. pp. 27-41. Springer (2017)

3. Aynulin, R.: Efficiency of transformations of proximity measures for graph clustering. In: International Workshop on Algorithms and Models for the Web-Graph. pp. 16-29. Springer (2019)

4. Aynulin, R.: Impact of network topology on efficiency of proximity measures for community detection. In: International Conference on Complex Networks and Their Applications. pp. 188-197. Springer (2019)

5. Barber, M.J., Clark, J.W.: Detecting network communities by propagating labels under constraints. Physical Review E 80(2), 026129 (2009)

6. Blondel, V.D., Guillaume, J.L., Lambiotte, R., Lefebvre, E.: Fast unfolding of communities in large networks. Journal of Statistical Mechanics: Theory and Experiment 2008(10), P10008 (2008)

7. Borg, I., Groenen, P.J.F.: Modern Multidimensional Scaling: Theory and Applications. Springer Science \& Business Media (2005)

8. Buckley, F., Harary, F.: Distance in Graphs. Addison-Wesley (1990)

9. Chebotarev, P.: Studying new classes of graph metrics. In: International Conference on Geometric Science of Information. pp. 207-214. Springer (2013)

10. Chebotarev, P., Shamis, E.: On the proximity measure for graph vertices provided by the inverse Laplacian characteristic matrix. In: Abstracts of the Conference "Linear Algebra and its Applications". pp. 6-7. University of Manchester, Manchester, UK (1995)

11. Chebotarev, P., Shamis, E.: On a duality between metrics and $\Sigma$-proximities. Automation and Remote Control 59(4), 608-612 (1998)

12. Chebotarev, P., Shamis, E.: On proximity measures for graph vertices. Automation and Remote Control 59(10), 1443-1459 (1998)

13. Chung, F.: The heat kernel as the pagerank of a graph. Proceedings of the National Academy of Sciences 104(50), 19735-19740 (2007)

14. Chung, F., Yau, S.T.: Coverings, heat kernels and spanning trees. Journal of Combinatorics 6, 163-184 (1998)

15. Chung, F.R.K.: Spectral Graph Theory, vol. 92. American Mathematical Soc. (1997)

16. Courtain, S., Leleux, P., Kivimäki, I., Guex, G., Saerens, M.: Randomized shortest paths with net flows and capacity constraints. Information Sciences (2020), to appear

17. Enright, A.J., Van Dongen, S., Ouzounis, C.A.: An efficient algorithm for largescale detection of protein families. Nucleic Acids Research 30(7), 1575-1584 (2002)

18. Estrada, E., Hatano, N.: Statistical-mechanical approach to subgraph centrality in complex networks. Chemical Physics Letters 439(1-3), 247-251 (2007)

19. Estrada, E., Hatano, N.: Communicability in complex networks. Physical Review E 77(3), 036111 (2008)

20. Estrada, E., Silver, G.: Accounting for the role of long walks on networks via a new matrix function. Journal of Mathematical Analysis and Applications 449(2), 1581-1600 (2017) 
21. Fortunato, S., Barthelemy, M.: Resolution limit in community detection. Proceedings of the National Academy of Sciences 104(1), 36-41 (2007)

22. Fotouhi, B., Momeni, N., Allen, B., Nowak, M.A.: Evolution of cooperation on large networks with community structure. Journal of the Royal Society Interface 16(152), 20180677 (2019)

23. Fouss, F., Francoisse, K., Yen, L., Pirotte, A., Saerens, M.: An experimental investigation of kernels on graphs for collaborative recommendation and semisupervised classification. Neural Networks 31, 53-72 (2012)

24. Fouss, F., Saerens, M., Shimbo, M.: Algorithms and Models for Network Data and Link Analysis. Cambridge University Press (2016)

25. Fouss, F., Yen, L., Pirotte, A., Saerens, M.: An experimental investigation of graph kernels on a collaborative recommendation task. In: Sixth International Conference on Data Mining (ICDM'06). pp. 863-868. IEEE (2006)

26. Göbel, F., Jagers, A.A.: Random walks on graphs. Stochastic Processes and Their Applications 2(4), 311-336 (1974)

27. Gösgens, M., Prokhorenkova, L., Tikhonov, A.: Systematic analysis of cluster similarity indices: Towards bias-free cluster validation. arXiv preprint arXiv:1911.04773 (2019)

28. Guex, G., Courtain, S., Saerens, M.: Covariance and correlation kernels on a graph in the generalized bag-of-paths formalism. arXiv preprint arXiv:1902.03002 (2019)

29. Guex, G., Kivimäki, I., Saerens, M.: Randomized optimal transport on a graph: framework and new distance measures. arXiv preprint arXiv:1806.03232 (2018)

30. Holland, P.W., Laskey, K.B., Leinhardt, S.: Stochastic blockmodels: First steps. Social Networks 5(2), 109-137 (1983)

31. Hubert, L., Arabie, P.: Comparing partitions. Journal of Classification 2(1), 193 $218(1985)$

32. Ivashkin, V., Chebotarev, P.: Do logarithmic proximity measures outperform plain ones in graph clustering? In: International Conference on Network Analysis. pp. 87-105. Springer (2016)

33. Jacobsen, K.A., Tien, J.H.: A generalized inverse for graphs with absorption. Linear Algebra and its Applications 537, 118-147 (2018)

34. Kandola, J., Cristianini, N., Shawe-Taylor, J.S.: Learning semantic similarity. In: Advances in Neural Information Processing Systems. pp. 673-680 (2003)

35. Katz, L.: A new status index derived from sociometric analysis. Psychometrika 18(1), 39-43 (1953)

36. Kirkland, S.J., Neumann, M.: Group Inverses of M-matrices and Their Applications. CRC Press (2012)

37. Kivimäki, I., Shimbo, M., Saerens, M.: Developments in the theory of randomized shortest paths with a comparison of graph node distances. Physica A: Statistical Mechanics and its Applications 393, 600-616 (2014)

38. Lancichinetti, A., Fortunato, S., Radicchi, F.: Benchmark graphs for testing community detection algorithms. Physical Review E 78(4), 046110 (2008)

39. Leleux, P., Courtain, S., Guex, G., Saerens, M.: Sparse randomized shortest paths routing with tsallis divergence regularization. arXiv preprint arXiv:2007.00419 (2020)

40. Lloyd, S.: Least squares quantization in pcm. IEEE Transactions on Information Theory 28(2), 129-137 (1982)

41. Luxburg, U.V., Radl, A., Hein, M.: Getting lost in space: Large sample analysis of the resistance distance. In: Advances in Neural Information Processing Systems. pp. 2622-2630 (2010) 
42. MacQueen, J.: Some methods for classification and analysis of multivariate observations. In: Proceedings of the Fifth Berkeley Symposium on Mathematical Statistics and Probability. vol. 14, pp. 281-297. Oakland, CA, USA (1967)

43. Mika, S., Ratsch, G., Weston, J., Scholkopf, B., Mullers, K.R.: Fisher discriminant analysis with kernels. In: Neural Networks for Signal Processing IX: Proceedings of the 1999 IEEE Signal Processing Society Workshop. pp. 41-48. IEEE (1999)

44. Newman, M.E.J., Girvan, M.: Finding and evaluating community structure in networks. Physical Review E 69(2), 026113 (2004)

45. Page, L., Brin, S., Motwani, R., Winograd, T.: The PageRank citation ranking: Bringing order to the web. Tech. rep., Stanford InfoLab (1999)

46. Pasta, M.Q., Zaidi, F.: Topology of complex networks and performance limitations of community detection algorithms. IEEE Access 5, 10901-10914 (2017)

47. Prokhorenkova, L.: Using synthetic networks for parameter tuning in community detection. In: International Workshop on Algorithms and Models for the WebGraph. pp. 1-15. Springer (2019)

48. Raghavan, U.N., Albert, R., Kumara, S.: Near linear time algorithm to detect community structures in large-scale networks. Physical Review E 76(3), 036106 (2007)

49. Shawe-Taylor, J., Cristianini et al., N.: Kernel Methods for Pattern Analysis. Cambridge University Press (2004)

50. Sommer, F., Fouss, F., Saerens, M.: Comparison of graph node distances on clustering tasks. In: International Conference on Artificial Neural Networks. pp. 192-201. Springer (2016)

51. Sommer, F., Fouss, F., Saerens, M.: Modularity-driven kernel k-means for community detection. In: International Conference on Artificial Neural Networks. pp. 423-433. Springer (2017)

52. Van Dongen, S.M.: Graph Clustering by Flow Smulation. Ph.D. thesis, Utrecht University (2000)

53. Von Luxburg, U.: A tutorial on spectral clustering. Statistics and Computing 17(4), 395-416 (2007)

54. Yen, L., Fouss, F., Decaestecker, C., Francq, P., Saerens, M.: Graph nodes clustering based on the commute-time kernel. In: Pacific-Asia Conference on Knowledge Discovery and Data Mining. pp. 1037-1045. Springer (2007)

55. Yen, L., Fouss, F., Decaestecker, C., Francq, P., Saerens, M.: Graph nodes clustering with the sigmoid commute-time kernel: A comparative study. Data \& Knowledge Engineering 68(3), 338-361 (2009)

56. Yen, L., Saerens, M., Mantrach, A., Shimbo, M.: A family of dissimilarity measures between nodes generalizing both the shortest-path and the commute-time distances. In: Proceedings of the 14th ACM SIGKDD International Conference on Knowledge Discovery and Data Mining. pp. 785-793 (2008) 